\title{
Evaluación Inicial del Seguro Popular sobre el Gasto Catastrófico en Salud en México
}

\section{Seguro Popular's initial evaluation of household catastrophic health spending in Mexico}

\author{
Jinneth Hernández-Torres ${ }^{1}$, Leticia Avila-Burgos ${ }^{2}$, AtanacioValencia-Mendoza ${ }^{2}$ y \\ Ofelia Poblano-Verástegui ${ }^{3}$
}

1 Hospital Rafael Uribe Uribe. Bogotá, Colombia. jinnether@hotmail.com

2 Instituto Nacional de Salud Pública de México. Cuernavaca, México.lavila@insp.mx;

avalencia@insp.mx.

3 Secretaria de Salud de México. Dirección General de Calidad y Educación en Salud. México

DF. ofeliapoblano@salud.gob.mx

Recibido 22 Marzo 2007/Enviado para Modificación 18 Noviembre 2007/Aceptado 25 Enero 2008

\section{RESUMEN}

Objetivo Estimar el resultado inicial del Seguro Popular de Salud (SPS) sobre el gasto catastrófico en salud (GC) de los hogares. Adicionalmente se estimó la relación de otros factores de importancia sobre el GC.

Material y métodos Estudio transversal a partir de la Encuesta de Evaluación del Seguro Popular levantada en los estados de Colima y Campeche en el segundo semestre del año 2002. El estudio se llevó a cabo en el primer semestre del año 2005. Se estimó primero la relación del SPS y otras covariables con el GC mediante un modelo probit. Después se estimó nuevamente la relación de las covariables y el GC tomando en cuenta la endogeneidad del GC con la afiliación al SPS, utilizando para ello un modelo probit bivariado. Finalmente, se realizaron algunas simulaciones para ver con más detalle la influencia de la utilización por tipo de servicio sobre el GC.

Resultados Para los hogares afiliados al SPS la probabilidad de incurrir en GC fue casi $8 \%$ menor, en comparación con los hogares no afiliados, controlando por las demás covariables y se corrigió por la endogeneidad. La probabilidad de GC siempre fue menor para los afiliados, independientemente del tercil económico al que pertenecían y del tipo de servicio utilizado.

Conclusiones Los resultados del estudio sugieren que el SPS está protegiendo financieramente a los hogares; sin embargo, aún constituye un reto llegar a la meta de reducción del $75 \%$ del GC.

Palabras Clave: Inversiones en salud, Seguridad Social, Sistemas de salud,México (fuente: DeCS, BIREME). 


\section{ABSTRACT}

Objective Estimating Seguro Popular de Salud's (SPS) initial outcome regarding households' catastrophic health spending (CHS). The relationship of other important factors to the CE was also estimated.

Material and methods A cross-sectional study, based on evaluating Seguro Popular's survey, was carried out in the Mexican states of Colima and Campeche during 2002; it was carried out during the first semester of 2005. SPS and other covariables' relationship with CHS was estimated by using the probit model. Such relationship was then estimated again using the bi-probit model, but taking endogeneity between CHS and SPS affiliation into consideration. Some simulations led to a detailed analysis of the influence of the use by type of service on the CHS.

Results The probability of SPS-affiliated households incurring $\mathrm{CHS}$ was about $8 \%$ less than un-affiliated households (controlled for other co-variables and corrected for endogeneity). The probability of incurring $\mathrm{CHS}$ was always less for affiliated people, independently of the income bracket which they belonged to and the kind of services used.

Conclusions The results suggested that SPS is financially protecting households; nevertheless, the goal of a $75 \%$ reduction in $\mathrm{CHS}$ has still to be achieved.

Key Words: Investments, Social security, health system, Mexico (source: MeSH, NLM).

$\mathrm{P}$ ara el año 2004 el gasto total en salud como porcentaje del Producto Interno Bruto (PIB) en México fue de 5,8 \%. De éste, 53,6 \% fue gasto privado, que en $94 \%$ provino del gasto de bolsillo de los hogares. Para el período 1992-2002 se estimó que para 3 a 4 \% de los hogares mexicanos este gasto fue de carácter catastrófico (1), entendiendo por éste una proporción mayor o igual a $30 \%$ del consumo disponible del hogar, que se define como el consumo total menos el consumo en alimentos (2).

El gasto catastrófico en salud (GC) tiene una mayor incidencia en los hogares más pobres, especialmente entre los que no tienen ningún aseguramiento en salud, quienes llegan a destinar en promedio $10 \%$ de su consumo disponible (3). En el año 2004, 1,8 \% de los hogares se empobrecieron por incurrir en un gasto en salud, es decir, que cruzaron la línea de pobreza o cayeron más abajo de ésta; de este porcentaje casi la totalidad pertenecía al quintil de ingreso más pobre. Entonces si se considera para ese mismo año, el porcentaje de hogares que incurrieron en GC o en gasto empobrecedor o en ambos, denominados gastos excesivos, estos representaron 4,1\%. Este tipo de gasto afectó al 9,7 $\%$ de la población del primer quintil $(4,5)$. El 60 \% de este GC en los hogares pobres se efectúa en atención ambulatoria y medicamentos (1). 
También se ha documentado que gastan más los hogares que: a) residen en zona rural; b) cuentan con personas mayores de 60 años de edad o niños; c) en donde el jefe del hogar es una mujer, cuando éste sobrepasa los 60 años de edad o cuando tiene bajo nivel educativo; d) cuentan con un miembro discapacitado, y e) enfrentan un parto $(1,6,7)$.

Tener que incurrir en gasto de bolsillo se convierte en una barrera para el acceso y la utilización de los servicios de salud. Así, se ha estimado que 20\% de la población mexicana tiene dificultades para pagar la atención y $25 \%$ la pospone por este motivo, esto es más frecuente entre los más pobres, los ancianos y quienes habitan en zona rural (8).

Los gastos de bolsillo y catastrófico reflejan, en parte, la falta de una adecuada garantía al acceso a los servicios de salud. Al respecto, algunos países han puesto en marcha distintas reformas a sus esquemas de financiamiento mediante esquemas de aseguramiento en salud, con la introducción del prepago como mecanismo para agrupar los riesgos financieros a los que puede conducir la demanda por servicios de salud, y en donde se focalizan subsidios para los más pobres, con el fin de eliminar las barreras económicas en el acceso a los servicios, aumentar la cobertura, disminuir el gasto de bolsillo, lo cual se conoce en su conjunto como protección financiera en salud (9).

Como parte de este esquema, México creó el Sistema de Protección Social en Salud (SPSS) con la reforma a la Ley General de Salud que entró en vigencia en el año 2004 con la operación del Seguro Popular de Salud (SPS), pero comenzó como un proyecto piloto en el año 2001 en cinco estados de la República: Aguascalientes, Colima, Campeche, Jalisco y Tabasco. Este es un seguro público de carácter voluntario dirigido a familias sin acceso a otros sistemas de seguridad social independientemente de su nivel socioeconómico. Este seguro ofrece un paquete de servicios definido a través de el Catálogo Universal de Servicios de Salud (CAUSES). El financiamiento es tripartito, proveniente de los recursos federales y estatales como un subsidio a la demanda, así como de los aportes anticipados de la población, según su nivel de ingreso, de las que están exentos los dos primeros deciles (10).

Con este nuevo esquema se pretende dar cobertura a la totalidad de la población sin acceso a la seguridad social para el año 2010, y reducir en 75\% el gasto catastrófico de la población a la que va dirigido el seguro (10). Hasta el año 2006 el SPS había alcanzado una cobertura de 5,1 millones de familias, lo que representa $43 \%$ de la población objetivo (11). 
Para el año 2005, en el estado de Colima se había alcanzado 100 \% de la afiliación de la población de los primeros dos deciles. En este estado desde el inicio de este programa se implantó la cuota cero para los hogares de los dos primeros deciles, se amplió el paquete de beneficios de 78 a 110 acciones en salud y de 165 a 221 medicamentos (12). Es importante señalar que éste fue uno de los estados de la Federación con gasto catastrófico más bajo, (2,2 \% de los hogares) para el año 2002 (1). Por su parte, en Campeche para este mismo período 2,5\% de los hogares incurrieron en gasto catastrófico y hasta el primer trimestre del año 2005 se había afiliado al SPS al 40 \% de la población objetivo $(1,12)$.

Dado que hay poca información publicada sobre el monitoreo del resultado que ha tenido la introducción del SPS en la reducción del gasto de bolsillo y catastrófico, este trabajo tiene como objetivo estimar el resultado inicial del SPS sobre el gasto catastrófico en salud en hogares afiliados en los estados de Colima y Campeche en el tercer trimestre del año 2002. Adicionalmente, se estima el efecto de otros factores de importancia asociados al gasto catastrófico en salud entre hogares afiliados y no afiliados al SPS en estos estados.

Marco conceptual

Se conoce como gasto de bolsillo en salud al total de las erogaciones que los hogares hacen para poder recibir la atención. Estas erogaciones incluyen tanto los gastos directamente relacionados con la atención, como aquellos que son necesarios para acceder a ésta (2).

En el análisis de la carga financiera que estas contribuciones representan para los hogares han sido planteados dos enfoques que pudieran ser complementarios. El primero define un gasto en salud como catastrófico cuando éste sobrepasa una proporción definida del ingreso o consumo disponibles del hogar, para lo cual se han utilizado diversos puntos de corte; éstos van desde 20 a 40\%, las limitaciones de este enfoque se mencionarán en la discusión $(9,13,14)$.

El segundo enfoque define un gasto en salud como empobrecedor cuando este desplaza a un hogar por debajo de la línea de pobreza (LP), o bien, profundiza su pobreza si es que ya se encontraba por debajo de esta línea. Este enfoque toma en cuenta cualquier monto de gasto en salud que hace que se empobrezcan los hogares independientemente de si sobrepasa o no la definición de GC (15). El problema de este enfoque radica en la medición misma de lo que es pobreza y, por lo tanto, en qué nivel ubicar la línea de pobreza (16). 
Tomando en cuenta que uno de los objetivos principales del SPS es la reducción del gasto catastrófico en salud, en este estudio nos centraremos en su análisis. Consideramos que los hogares pueden incurrir en gasto en salud una vez que han cubierto un mínimo de necesidades básicas de subsistencia, que para este estudio se consideró como cubrir los gastos de alimentación.

\section{MATERIAL Y MÉTODOS}

Este estudio se basa en un análisis secundario y transversal de la Encuesta de Evaluación del Seguro Popular (EESP), llevada a cabo en los estados de Colima y Campeche en septiembre del año 2002 por el Grupo de Evaluación Externa del SPS, conformado por el Instituto Nacional de Salud Pública de México y FUNSALUD, con el fin de evaluar la fase piloto.

Para la EESP se seleccionaron 1120 hogares afiliados al SPS mediante un muestreo aleatorio simple de los listados de hogares que fueron incluidos en la evaluación inicial o basal realizada tres meses antes en los estados de Colima y Campeche. Se consideraron como casos a los hogares que en la medición basal de la evaluación, se identificaron como afiliados al SPS. El grupo control se conformó por dos hogares por cada caso entre los hogares que en la medición basal reportaron no contar con seguridad social y que al momento de la segunda evaluación (la realizada para estudio) no presentaron cambios en su calidad de afiliación a la seguridad social y que además residieran en el mismo barrio del control, lo que dio un total de 232 hogares no afiliados. Asimismo, el cálculo de la muestra se hizo considerando que un $10 \%$ de estos hogares incurrían en GC, dado que era población sin seguridad social y ubicada en los menores deciles de ingreso.

La submuestra para este estudio quedó conformada por aquellos hogares que contaban con mínimo tres meses de afiliación al SPS previos al levantamiento de la encuesta de evaluación, así como todos los hogares sin afiliación a la seguridad social. Otros criterios de inclusión fueron el de incorporar solamente aquellos hogares que reportaron haber tenido al menos un integrante con algún problema de salud, embarazo, que requirió atención de parto, o bien, que utilizó servicios preventivos en los últimos tres meses. Así, la submuestra para este estudio estuvo conformada por 2158 hogares, de los cuales 482 estaban afiliados al SPS y 1676 no contaron con ninguna afiliación. La unidad de análisis del estudio fue el hogar, definido como el número de personas que comparten un mismo gasto alimentario. 
En este estudio se definió como gasto de bolsillo en salud a cualquier desembolso realizado al momento de recibir servicios de salud tanto formales como no formales. El gasto catastrófico (GC) se definió como una variable en forma dicotómica, y se consideró cuando un hogar destinó 30 \% del consumo disponible a la atención a la salud, o para acceder a ésta, lo que es congruente con lo que se recomienda para los países de ingreso medio como México $(1,3)$. Este gasto se calculó de forma trimestral acorde con la temporalidad cuando la encuesta fue levantada. El consumo disponible se estimó como el consumo o gasto total reportado por el hogar, menos el gasto en alimentos, excluyendo de este último el gasto en bebidas alcohólicas o alimentos fuera del hogar (2).

Las variables independientes para predecir GC se agruparon en cinco categorías de factores:

1. Condición de afiliación del jefe o cónyuge al SPS ; 2 . Entidad federativa de residencia (Colima o Campeche) ; 3. Características y composición del hogar (características del jefe del hogar: edad, actividad laboral), tamaño del hogar e índice de dependencia (cociente de la suma de integrantes del hogar menores de 5 y mayores de 60 años de edad entre el total de personas en el hogar) ; 4. Estatus económico del hogar (número de perceptores de ingresos, consumo disponible trimestral per cápita-CDPC) ; 5. Necesidad de atención en salud del hogar (tipo de problema de salud ambulatorio en los últimos tres meses, grado de severidad auto-percibida, tipo de servicio de salud utilizado, institución en la que se utilizó el servicio, presencia de enfermos crónicos, y presencia de discapacitados) (19).

Para caracterizar a la población del estudio y comparar el gasto de bolsillo y catastrófico por rubros se realizó inicialmente un análisis univariado con medidas de tendencia central y se probó la normalidad de las variables. El análisis bivariado se realizó con pruebas de $j i$ cuadrada, para comparación de proporciones, y pruebas de Mann-Whitney, para comparación de rangos de las variables con distribución no normal.

Construcción del modelo multivariado

En este trabajo se probaron dos modelos para estimar el resultado inicial del SPS sobre el gasto catastrófico de los hogares. El primero fue un modelo probit en el que se incluyeron las variables que en el análisis bivariado tuvieron un valor de $p<0,025$, o bien, aquellas que por su relevancia teórica deberían incluirse en el modelo. El segundo fue un modelo probit bivariado, el cual permite probar la existencia o no de un sesgo de selección del SPS y corregirlo 
en caso de existir (17). En términos econométricos el sesgo de selección es equivalente a la existencia de endogeneidad entre la variable de afiliación al SPS y el GC, de forma que pueden existir una serie de factores no observables, no medibles o no incluidos de manera explícita en el modelo que influyen al mismo tiempo sobre afiliación al SPS y sobre el GC, en presencia de la cual se obtiene una estimación sesgada de la asociación entre SPS y GC si ésta no se toma en cuenta.

Como prueba de la existencia o no de endogeneidad, y poder elegir así entre un modelo probit y un probit bivariado se estimó la correlación de los términos de error de las dos ecuaciones estimadas de forma simultánea en el modelo probit bivariado: la de GC y la de afiliación al SPSy como prueba adicional la diferencia en el signo para el coeficiente de la variable de afiliación al SPS entre los dos modelos. En la identificación de variables instrumentales (Z) para explicar la variable endógena de afiliación al SPS y que no deben estar relacionadas con el GC, se usó el método de la razón de verosimilitud, mediante una prueba $j i$ cuadrada aplicada a los dos modelos estimados para predecir GC, en uno de los cuales se incluyeron las variables Z (18). Después de probar varios conjuntos de variables identificadoras, se encontró al sexo, nivel de escolaridad del jefe del hogar, así como la presencia de alguna embarazada entre las integrantes del hogar, como un conjunto adecuado de variables Z.

Finalmente, a partir del modelo probit bivariado se realizaron simulaciones discriminando por afiliación al SPS y por tercil de CDPC, con el fin de estimar la probabilidad de GC variando la entidad federativa y si un hogar sólo utilizará un tipo de servicios. El análisis se llevó a cabo con el paquete estadístico Stata 8.2 .

\section{RESULTADOS}

Los hogares afiliados al SPS tuvieron en promedio un menor nivel de escolaridad del jefe y de consumo disponible per cápita en relación con los hogares no afiliados (Tabla 1). Asimismo, se encontró que los hogares afiliados tuvieron significativamente una mayor proporción de embarazadas y de utilización para todos los tipos de servicios de salud, especialmente para la atención del parto, excepto para los servicios preventivos del niño; esta utilización se realizó principalmente en el sector público (Tabla 2). 
Tabla 1. Características sociodemográficas de los hogares de la Encuesta de Evaluación del Seguro Popular, en los estados de Colima y Campeche México, 2002

\begin{tabular}{|c|c|c|c|}
\hline Variables & $\begin{array}{c}\% \text { Hogares } \\
\text { No Afiliados } \\
N=1676\end{array}$ & $\begin{array}{c}\text { \%Hogares } \\
\text { afiliados } \\
\mathrm{N}=482\end{array}$ & p \\
\hline \multicolumn{4}{|l|}{ Estado } \\
\hline Colima & 75,5 & 64,3 & \\
\hline Campeche & 24,5 & 35,6 & \\
\hline \multicolumn{4}{|l|}{ \#Personas en el hogar } \\
\hline $1-2$ & 16,4 & 14,7 & \multirow{3}{*}{$0.71 \dagger$} \\
\hline $3-4$ & 43,7 & 43,1 & \\
\hline 5-6 & 27,6 & 28,6 & \\
\hline $7-13$ & 12,1 & 13,4 & \\
\hline Indice de dependencia & 0.2 & 0,2 & $0.72^{\circ}$ \\
\hline \multicolumn{4}{|l|}{ Genero jefe hogar } \\
\hline Hombre & 79,3 & 76,9 & \multirow[t]{2}{*}{$0.25 \dagger$} \\
\hline Mujer & 20,6 & 23,0 & \\
\hline \multicolumn{4}{|l|}{ Edad jefe hogar } \\
\hline $17-40$ & 49,8 & 45,4 & \multirow{3}{*}{$0.20 \dagger$} \\
\hline $41-60$ & 35,8 & 38,1 & \\
\hline 61 y mas & 14,3 & 16,3 & \\
\hline \multicolumn{4}{|c|}{ Nivel de escolaridad jefe de hogar* } \\
\hline Ninguno & 13,8 & 13,5 & \multirow{4}{*}{$0.00 \dagger$} \\
\hline Primaria & 51 & 60,9 & \\
\hline Secundaria & 21,3 & 18,3 & \\
\hline Preparatoria o más & 13,8 & 7,2 & \\
\hline \multicolumn{4}{|c|}{ Actividad laboral jefe de hogar } \\
\hline Desempleado & 4 & 3,3 & \multirow{5}{*}{$0.16 \dagger$} \\
\hline Trabajador & 76,9 & 72,8 & \\
\hline No trabaja/Estudiante & 9,5 & 12,2 & \\
\hline Pensionado/incapacitado & 1,5 & 2,4 & \\
\hline Hogar & 7,9 & 9,1 & \\
\hline Perceptores de ingresos & 1 & 1 & $0.18^{-}$ \\
\hline \multicolumn{4}{|c|}{ Consumo disponible per capita $x$ tercil ${ }^{*}$} \\
\hline I:4-399.352 & 28,8 & 35,8 & \multirow{3}{*}{$0.00 \dagger$} \\
\hline II:399.353-1034.76 & 32,7 & 35,4 & \\
\hline III:1034.77-1503765 & 38,4 & 28,6 & \\
\hline
\end{tabular}

El gasto en servicios ambulatorios y preventivos representó la mayor proporción del CDPC y ésta fue significativamente mayor en los hogares afiliados (10,8), en comparación con los no afiliados $(3,6)$. En contraste, el gasto en hospitalización fue significativamente mayor para los hogares no afiliados $(16,2)$ que para los afiliados (0) y representó, en promedio, la mayor proporción del CDPC. Pese a estas diferencias, al analizar las medianas del gasto de bolsillo total per cápita no se encontraron diferencias estadísticamente significativas entre ambos tipos de hogares. El porcentaje de hogares que incurrieron en GC fue de $27 \%$ entre los hogares no afiliados y de $20 \%$ entre los hogares afiliados al SPS, aunque esta diferencia no fue significativa. 
Tabla 2. Características de necesidad y utilización de los hogares de la Encuesta de Evaluación del Seguro Popular, en los estados de Colima y CampecheMéxico, 2002

\begin{tabular}{|c|c|c|c|}
\hline $\begin{array}{c}\text { Factores de necesidad y } \\
\text { Utilización }\end{array}$ & $\begin{array}{l}\% \text { Hogares No } \\
\text { Afiliados }\end{array}$ & $\begin{array}{l}\% \text { Hogares } \\
\text { afiliados }\end{array}$ & $\mathrm{P}$ \\
\hline \multicolumn{4}{|l|}{ Problema salud ambulatorio } \\
\hline Ninguno & 42,6 & 40,8 & \multirow{4}{*}{$0.64 \dagger$} \\
\hline Agudo & 31,8 & 30,9 & \\
\hline Crónico & 2 & 2,7 & \\
\hline Otras y combinación & 23,4 & 25,5 & \\
\hline Severidad problema ambulatorio & $\mathrm{N}=961$ & $\mathrm{~N}=285$ & \multirow{4}{*}{$0.32 \dagger$} \\
\hline Leve & 37,7 & 36,4 & \\
\hline Moderado & 29,2 & 25,9 & \\
\hline Severo y combinación & 32,9 & 37,5 & \\
\hline \multicolumn{4}{|l|}{ Embarazo* } \\
\hline No & 93,7 & 90,4 & \multirow[t]{2}{*}{$0.01 \dagger$} \\
\hline $\mathrm{Si}$ & 6,3 & 9,5 & \\
\hline Motivo de hospitalización & $\mathrm{N}=72$ & $\mathrm{~N}=36$ & \multirow{4}{*}{$0.80 \dagger$} \\
\hline Cirugia & 82,1 & 77,7 & \\
\hline Otras & 16,4 & 19,4 & \\
\hline Cirugia y otras & 1,3 & 2,7 & \\
\hline \multicolumn{4}{|l|}{ Personas discapacitadas } \\
\hline No & 90,5 & 89 & \multirow[t]{2}{*}{$0.32 \dagger$} \\
\hline $\mathrm{Si}$ & 9,4 & 11 & \\
\hline \multicolumn{4}{|l|}{ Enfermos crónicos } \\
\hline No & 53,3 & 52,9 & \multirow[t]{2}{*}{$0.86 \dagger$} \\
\hline $\mathrm{Si}$ & 46.6 & 47,1 & \\
\hline Utilización preventiva* & $\mathrm{N}=1676$ & $\mathrm{~N}=482$ & \multirow{6}{*}{$0.00 \dagger$} \\
\hline Ninguna & 50,1 & 39,4 & \\
\hline Niño & 17 & 15,7 & \\
\hline Reproductiva & 9,9 & 14,1 & \\
\hline Detección crónicas & 5,6 & 6,8 & \\
\hline Otras u combinación & 17,1 & 23,8 & \\
\hline Utilización ambulatoria (\# & $N=1676$ & $\mathrm{~N}=482$ & \\
\hline personas) & 19,9 & 14 & \\
\hline 0 & 67,4 & 71,5 & $0.06+$ \\
\hline \multirow{2}{*}{\multicolumn{4}{|c|}{$2-10$}} \\
\hline & & & \\
\hline Utilización control prenatal* & $\mathrm{N}=105$ & $\mathrm{~N}=46$ & \multirow{3}{*}{$0.05+$} \\
\hline No & 15,2 & 4,3 & \\
\hline $\mathrm{Si}$ & 84,7 & 95,6 & \\
\hline \multicolumn{4}{|l|}{ Utilización atención parto* } \\
\hline No & 76,1 & 41,3 & \multirow[t]{2}{*}{$0.00 \dagger$} \\
\hline $\mathrm{Si}$ & 23,8 & 58,7 & \\
\hline \multicolumn{4}{|l|}{ Utilización hospitalización* } \\
\hline No & 95,6 & 92,5 & \multirow[t]{2}{*}{$0.00 \dagger$} \\
\hline $\mathrm{Si}$ & 4,3 & 7,4 & \\
\hline Dias de hospitalización & 3 & 3 & $0.80^{-}$ \\
\hline
\end{tabular}

Para la estimación de ambos modelos se introdujo el CDPC por tercil por no cumplir con el supuesto de linealidad en su forma continua, y la edad del jefe 
del hogar de forma cuadrática para captar el efecto no lineal. Se mantuvieron en los modelos la interacción entre la presencia de enfermos crónicos y la utilización de servicios de hospitalización por resultar significativa.

Con el modelo probit se obtuvo una estimación de signo positivo entre afiliación al SPS y GC (1,6 \%) lo que resultaría contraintuitivo si no fuera por la existencia de endogenoeidad, ya que esto significaría que estar afiliado al SPS se asocia con una mayor posibilidad de incurrir en GC (Tabla 3). Este conjunto de evidencias, nos conduce al rechazo del modelo probit a favor del modelo probit bivariado, sobre el cual estaremos describiendo resultados y discutiendo conclusiones en lo sucesivo.

De acuerdo con el modelo probit bivariado se obtuvo que la probabilidad estimada de incurrir en GC para un hogar afiliado al SPS que tuvo necesidades de atención fue 7.7\% menor, comparado con un hogar no afiliado en las mismas condiciones de necesidad (Tabla 3). Una vez controlando por el resto de covariables incluidas en el modelo se encontró que los hogares del estado de Campeche tuvieron una probabilidad mayor de GC, en comparación con los del estado de Colima. A partir de las simulaciones se encontró que la probabilidad de incurrir en GC fue menor para los hogares afiliados al SPS en ambos estados, especialmente en los de Colima.

Con relación a las variables de composición del hogar se encontró que cada integrante adicional en el hogar disminuye la probabilidad de incurrir en GC, ajustando por las demás covariables. Por el contrario, si el número de menores de cinco o mayores de 60 años de edad aumenta, la probabilidad de incurrir en GC aumenta también. En relación con el número de perceptores de ingreso, como se esperaba, por cada incremento en el número de éstos disminuye la probabilidad de incurrir en GC.

La edad del jefe del hogar se relacionó de forma positiva con el GC, pero de forma decreciente. Los hogares cuyo jefe era empleado tuvieron una probabilidad menor de GC, comparados con los que se dedicaban a una actividad no remunerada. Asimismo, los hogares del tercil más pobre del CDPC tuvieron una probabilidad mayor de GC, comparados con los del tercil más rico. Como se esperaba, el tipo de necesidad y utilización de servicios de salud estuvieron positivamente relacionados con el GC.

A partir de las simulaciones se encontró que la probabilidad de incurrir en GC para ambos tipos de hogares fue en orden de importancia debido a: 1) la 
utilización de servicios de hospitalización; 2) la utilización de servicios de control prenatal; 3) la utilización de servicios de parto; 4) la utilización de servicios ambulatorios, y 5) y la presencia de enfermos crónicos (Figura 2). Adicionalmente, la probabilidad de incurrir en GC fue siempre mayor para los hogares más pobres independientemente de su estatus de afiliación.

Tabla 3. Modelos econométricos de Gasto Catastrófico en hogares de la Encuesta de Evaluación del Seguro Popular, en los Estados de Colima y Campeche México, 2002

\begin{tabular}{|c|c|c|c|c|c|c|}
\hline \multirow[t]{2}{*}{ Variables } & \multicolumn{3}{|c|}{ Modelo probit } & \multicolumn{3}{|c|}{ Modelo probit bivariado } \\
\hline & Coef. & $\mathrm{P}>|\mathrm{z}|$ & EM \% & Coef. & $\mathrm{P}>|\mathrm{z}|$ & $\mathrm{EM} \%$ \\
\hline Seguro popular $(1=\mathrm{Si} ; 0=\mathrm{No})^{*}$ & 0.06 & 0.44 & 1,6 & -0.87 & 0.02 & $-7,7$ \\
\hline Estado $(1=\text { Campeche; Colima }=0)^{*}$ & 0.29 & 0 & 8.1 & 0.36 & 0.00 & 7,1 \\
\hline \multicolumn{7}{|l|}{ Composición Hogar } \\
\hline Taman̆o* & -0.10 & 0 & $-14,8$ & -0.08 & 0.00 & $-6,3$ \\
\hline Indice de dependencia" & 0.60 & 0 & 20,2 & 0.55 & 0.00 & 11,4 \\
\hline Edad jefe. & 0.02 & 0.06 & 3,9 & 0.04 & 0.01 & 7,4 \\
\hline Edad jefe2* & 0.00 & 0.04 & 0,0 & 0.00 & 0.01 & $-0,1$ \\
\hline \multicolumn{7}{|l|}{ Actividad Jefe* } \\
\hline No remunerada & 1 & - & - & - & - & - \\
\hline Trabajo & -0.14 & 0.08 & -4.0 & -0.16 & 0.06 & $-1,8$ \\
\hline Pensionado/incapacitado & -0.05 & 0.82 & $-1,5$ & 0.04 & 0.88 & 2,8 \\
\hline \multicolumn{7}{|l|}{ Nivel Socioeconómico Hogar } \\
\hline \multicolumn{7}{|l|}{ Consumo disponible $\times$ tercil* } \\
\hline III & 1 & - & - & - & - & - \\
\hline II & 0.16 & 0.05 & 4,5 & 0.21 & 0.01 & 3,5 \\
\hline i & 0.88 & 0 & 26,1 & 0.84 & 0.00 & 9,2 \\
\hline Perceptores ingreso: & -0.03 & 0.43 & $-5,0$ & -0.06 & 0.15 & $-15,0$ \\
\hline \multirow{2}{*}{\multicolumn{7}{|c|}{ Necesidad atención salud Hogar }} \\
\hline Severidad problema salud* & & & & & & \\
\hline Ninguna, leve moderada & 1 & - & - & - & - & - \\
\hline Severa y combinación & 0.40 & 0 & 11,8 & 0.37 & 0.00 & 3,6 \\
\hline Enfermos crónicos $(1=\mathrm{Si} ; 0=\mathrm{No})^{*}$ & 0.34 & 0 & 8,9 & 0.32 & 0.00 & 3,3 \\
\hline Discapacidad $(1=\mathrm{Si} ; 0=\mathrm{No})^{*}$ & 0.02 & 0.85 & 0,5 & 0.03 & 0.82 & 0,7 \\
\hline \multicolumn{7}{|l|}{ Utilización control prenatal } \\
\hline$(1=\mathrm{Si} ; 0=\mathrm{No})^{*}$ & 0.70 & 0 & 22,9 & 0.75 & 0.00 & 6,9 \\
\hline Utilización parto $(1=\mathrm{Si} ; 0=\mathrm{No})^{*}$ & 0.49 & 0.03 & 15.4 & 0.45 & 0.03 & 4,4 \\
\hline \multicolumn{7}{|l|}{ Utilización hospitalización } \\
\hline$(1=\mathrm{Si} ; 0=\mathrm{No})^{*}$ & 1.52 & 0 & 54,2 & 1.38 & 0.00 & 10,5 \\
\hline \# Personas utilización ambulatoria* & 0.19 & 0 & 5,0 & 0.17 & 0.00 & 16,8 \\
\hline \multicolumn{7}{|l|}{ Enfermedad crónica y utilización } \\
\hline Hospitalización $(1=\mathrm{Si} ; 0=\mathrm{No})^{*}$ & -0.90 & 0.00 & $-14,9$ & -0.83 & 0.00 & $-6,8$ \\
\hline Constante & -1.89 & 0 & & -1.86 & 0 & \\
\hline N & 2127 & & & 2127 & & \\
\hline tho & & & & 0.5678 & & \\
\hline$p X^{2}$ & & & & 0.102 & & \\
\hline
\end{tabular}




\section{DISCUSIÓN}

El principal hallazgo del presente estudio sugiere que el SPS protegió a los hogares afiliados contra el gasto catastrófico en salud, lo cual es consistente con otros estudios realizados en México en los que se ha encontrado menor gasto de bolsillo y catastrófico entre quienes tienen algún aseguramiento en salud ,dentro de estos el SPS, comparados con los que no lo tienen, aunque dichos estudios no ajustaron por la endogeneidad de la afiliación a los esquemas de aseguramiento en salud con el GC $(1,3,4,6,19)$. Los resultados sugieren también que los hogares más pobres tienen mayor probabilidad de GC que los hogares con mayor consumo disponible, hallazgo que es consistente con los resultados de otros estudios en México antes de la implantación del SPS $(3,6)$. Pero un reciente estudio encontró que la reducción del gasto excesivo en salud entre los años 2000 y 2004 ha sido mayor para los hogares de los dos primeros quintiles y para los no asegurados o afiliados al SPS, pero sin poder atribuir el efecto a este último (4).

El estudio sugiere que en el estado de Colima los hogares, independientemente de su estatus de afiliación tuvieron una menor probabilidad de incurrir en GC que los del estado de Campeche. Futuros estudios podrían explorar si existen diferentes condiciones de oferta, disponibilidad y organización de los servicios de salud entre ambos estados que pudieran explicar este hallazgo.

La relación del resto de las variables con la probabilidad de GC refleja las características de un hogar que hacen que éste pueda asumir mejor la carga financiera que representa el gasto de bolsillo y son consistentes con lo reportado en otros estudios $(6,7,14)$. Solamente el tamaño del hogar tuvo una relación negativa con el GC, lo que no es consistente con los hallazgos reportados en estudios previos (14). Esto puede deberse a que un mayor número de personas puede estar relacionado con la generación de economías de escala en el hogar, una vez que se ha controlado por el número de dependientes económicos y perceptores de ingresos.

Es importante señalar que este estudio pudo subestimar el GC por los siguientes factores: a) por el punto de corte para definirlo, ya que para algunos hogares, especialmente los más pobres, la proporción del gasto en salud respecto al consumo disponible para ser considerado catastrófico puede ser menor (2) ; b) el utilizar el gasto alimentario como proxy para las necesidades de subsistencia de un hogar es una consideración reduccionista que sobreestima el consumo

disponible, y c) por disponibilidad de información no pudieron ser considerados 
algunos otros costos como los relacionados con el transporte durante el proceso de búsqueda de atención, por lo que el gasto de los hogares en salud puede estar también subestimado (15).

Hay que tener en cuenta que para la fecha de la encuesta el SPS tenía poco tiempo de operación, lo que puede considerarse un período de prueba o ajuste. Por tal motivo, es posible que la contribución de éste a la reducción del GC subestime el efecto que pudiera tener una vez que el SPS se encuentre operando con un tiempo suficiente de experiencia, y que la población conozca mejor la forma de acceder a él. Adicionalmente, el estudio no toma en cuenta otros factores de nivel macroeconómico o de pertenencia a programas contra la pobreza como Oportunidades que también podrían haber contribuido al efecto observado (4).

El hecho de que existiera mayor número de embarazadas entre los hogares afiliados al SPS y de que además requirieran atención de parto señala que ocurre un efecto de selección adversa por parte de los hogares para la afiliación al SPS; esto es característico de los seguros voluntarios debido a que los hogares con mayores necesidades en salud tienen más incentivos para afiliarse (12). Este fenómeno debe ser considerado para la viabilidad futura del SPS.

En conclusión, los resultados del estudio sugieren que el SPS está cumpliendo con uno de sus principales objetivos al proteger financieramente a los hogares y de esta forma contribuyendo a que los hogares por el gasto en salud, no tengan que hacer grandes recortes a su presupuesto destinado a otras necesidades. Sin embargo, sigue constituyendo un reto llegar a la meta de reducir en 75 \% el GC en la población objetivo. Lograrlo, implicará además de aumentar la afiliación, garantizar la cobertura efectiva del CAUSES, fortaleciendo la red prestadora de servicios de salud y disminuir otras barreras para el acceso a los servicios de salud, distintas a las financieras (3). Así como evaluar la inclusión en el CAUSES de la atención hospitalaria de enfermedades de alta prevalencia y muy alto costo, como la diabetes y enfermedades cardiovasculares. Lo anterior, cobra especial importancia ante fenómenos como el envejecimiento de la población y la transición epidemiológica.

Un segundo reto muy importante es continuar mejorando la equidad vertical en relación con el GC como uno de los componentes principales de la equidad financiera en el SPS. En este sentido, también será muy importante mantener la progresividad de los aportes anticipados, especialmente el régimen no contributivo para los hogares más pobres. Aunque hasta ahora no existen 
copagos, la Ley de Protección Social en Salud dejó abierta la posibilidad de cuotas moderadoras para evitar el riesgo moral, lo cual podría profundizar la distribución inequitativa del GC, así como imponer barreras financieras al acceso y afectar la afiliación al SPS, especialmente si se implementarán para los hogares más pobres *

Agradecimientos. A Gustavo Olaíz por permitir el uso de la base de datos, a Alonso Restrepo por la corrección de estilo y a mis padres y amigos por su apoyo de siempre.

\section{REFERENCIAS}

1. Sesma S, Pérez R, Gómez O. Síntesis Ejecutiva: Magnitud y origen de los gastos catastróficos por motivos de salud por entidad federativa: México 2002-2003. México,DF: Secretaría de Salud de México;2004.

2. Xu K. Household health system contributions and capacity to pay: Definitional, empirical, and technical challenges. En: Murray C, Evans D. Health System Performance Assesment. Washington, DC: Organización Mundial de la Salud; 2003.

3. Knaul F, Arreola H. El Sistema de Protección Social en Salud de México: efectos potenciales sobre la justicia financiera y los gastos catastróficos de los hogares. En: Funsalud. Caleidoscopio de la Salud. México, DF: Funsalud; 2003.

4. Knaul F, Arreola H, Méndez O. Protección financiera en salud: México, 1992 a 2004. Salud Pública Méx 2005;47:430-439.

5. Organización para la Cooperación y el Desarrollo Económico. Estudios de la OCDE sobre los Sistemas de Salud: México. México, DF: Organización para la Cooperación y el Desarrollo Económico; 2005.

6. Torres A, Knaul F. Determinantes del gasto de bolsillo en salud e implicaciones para el aseguramiento universal en México:1992-2000. En: Funsalud. Caleidoscopio de la Salud. México, DF: Funsalud; 2003.

7. Parker S, Wong R. Household income and health care expenditures in Mexico. Health Policy 1997; 40:237-255.

8. Arreola H, Soto H, Garduño J. Determinantes de la no demanda de atención a la salud en México. En: Funsalud Caleidoscopio de la Salud. México, DF: Funsalud; 2003.

9. Xu K, Evans D, Kawabata K, Zeramdini R, Klavus J, Murray C. Household catastrophic health expenditure: A multicountry analysis. The Lancet 2003; 362: 111-117.

10. Presidencia de la República. Reglamento de la Ley General de Salud en Materia de Protección social en Salud del 5 abril de 2004. Disponible en: http:// www.salud.gob.mx Consultado en: Mayo 2005.

11. Comisión Nacional de Protección Social en Salud. Informe de resultados segundo semestre 2006. México: Comisión Nacional de Protección Social en Salud; 2007. 
12. Secretaría de Salud, Instituto Nacional de Salud Pública. Evaluación del Seguro Popular. México, DF: SSA; 2002.

13. Murray C, Xu K. Assessing the distribution of household financial contributions to the health system: Concepts and empirical application. En: Murray C, Evans D. Health System Performance Assesment. Washington, DC: Organización Mundial de la Salud; 2003.

14. Wyszewianski L. Families with catastrophic health care expenditures. Health. Serv Res 1986;21(5):617-634.

15. Wagstaff A, Doorslaer E. Catastrophe and impoverishment in paying for health care: with applications to Vietnam 1993-1998. Health Econ. 2003; 12:921-34.

16. Boltvinik J. La pobreza en México. I. Metodologías y evolución. Salud Pública Mex 1995;37(4):288-297.

17. Greene W. Econometric analysis. Fourth Edition.Washignton:Prentice Hall;2000.

18. Bollen K, Guilkey D, Mroz T. Binary outcomes and endogenous explanatory variables: tests and solutions with an application to the demand for contraceptive use in Tunisia. Demography 1995; 32: 111-131.

19. Secretaría de Salud, Instituto Nacional de Salud Pública de México. Evaluación Seguro Popular Enero-Agosto;2003. México, DF: Secretaría de Salud de México; 2003. 